\section{Fungal Bloodstream Infections Associated with a Compounded Intravenous Medication at an Outpatient Oncology Clinic — New York City, 2016}

Amber M. Vasquez, MD ${ }^{1,2}$; Jason Lake, $\mathrm{MD}^{1,2}$; Stephanie Ngai, $\mathrm{MPH}^{3}$; Megan Halbrook, $\mathrm{MPH}^{3}$; Snigdha Vallabhaneni, MD ${ }^{4}$; M. Shannon Keckler, $\mathrm{PhD}^{2,5}$; Heather Moulton-Meissner, $\mathrm{PhD}^{2}$; Shawn R. Lockhart, $\mathrm{PhD}^{4}$; Christopher T. Lee, MD ${ }^{1,3}$; Kiran Perkins, $\mathrm{MD}^{2}$; Joseph F. Perz, $\mathrm{DrPH}^{2}$; Mike Antwi, $\mathrm{MD}^{3}$; Miranda S. Moore, $\mathrm{MPH}^{3}$; Jane Greenko, $\mathrm{MPH}^{6}$; Eleanor Adams, $\mathrm{MD}^{6}$; Janet Haas, $\mathrm{PhD}^{7}$; Sandra Elkind, $\mathrm{MS}^{7}$; Marjorie Berman, $\mathrm{MS}^{8}$; Dani Zavasky, $\mathrm{MD}^{7}$; Tom Chiller, $\mathrm{MD}^{4}$; Joel Ackelsberg, $\mathrm{MD}^{3}$

On May 24, 2016, the New York City Department of Health and Mental Hygiene notified CDC of two cases of Exophiala dermatitidis bloodstream infections among patients with malignancies who had received care from a single physician at an outpatient oncology facility (clinic A). Review of January 1May 31, 2016 microbiology records identified E. dermatitidis bloodstream infections in two additional patients who also had received care at clinic A. All four patients had implanted vascular access ports and had received intravenous (IV) medications, including a compounded IV flush solution containing saline, heparin, vancomycin, and ceftazidime, compounded and administered at clinic $\mathrm{A}$.

E. dermatitidis (previously known as Wangiella dermatitidis) is a neurotropic, dark pigment-forming fungus that is found in the environment (1). Health care-associated infections have been reported and include catheter-associated fungemia (1) and an outbreak of $E$. dermatitidis meningitis associated with contaminated injectable steroids prepared by a compounding pharmacy (2).

A case was defined as any non-Candida species yeast or mold identified on culture of blood or central venous catheter (CVC) (implanted port or peripherally inserted central catheter) from a patient who received care at clinic A during January 1-May 31, 2016. Case finding included microbiology record review, medical record review, and requests for screening blood and CVC cultures for all patients who had received an IV medication at clinic A during this period.

During January 1-May 31, 2016, a total of 153 patients were seen at clinic A, 38 (25\%) of whom received an IV medication. Among these 38 patients, six were deceased before the investigation began with case status undetermined, and three declined to be evaluated, leaving 29 patients for analysis. Seventeen $(59 \%)$ of the 29 patients met the case definition, including 13 whose cultures yielded $E$. dermatitidis, two with
Rhodotorula mucilaginosa, and two with both fungi. No cases were identified among patients who did not receive IV medications. Five of the 17 cases were identified in symptomatic patients who sought medical care for infection or underlying medical conditions, and the remaining 12 were identified by screening blood or CVC culture. Two of the infected patients died at 10 days and 12 weeks after positive culture, respectively; it was unclear whether the deaths were related to the infections.

The 17 patients with a positive culture (by blood or CVC culture) did not share a common chemotherapeutic exposure or a common adjunctive IV therapy (e.g., dexamethasone or ondansetron). However, all patients with or without a positive culture were exposed to the compounded IV flush solution. Patients with a positive culture received a median of 12 flushes (range $=2-20$ ) during the study period compared with a median of four flushes for those with a negative culture (range $=1-12)(\mathrm{p}=0.004$, Kruskal-Wallis test $)$.

Twenty-four of the 29 patients had a CVC, and five received IV medications through a peripheral line; all 17 patients with a positive culture had a CVC (attack rate of $71 \%$ compared with $0 \%$ among those with a peripheral line). After the screening blood culture was obtained, all patients with a CVC had their venous access removed and began 4-6 weeks of antifungal therapy. The five patients who received IV medications through a peripheral line had negative screening blood cultures, and antifungal treatment was not recommended.

Assessment of clinic A revealed failures to meet CDC infection control standards for outpatient oncology settings (3) as well as standards for sterile medication compounding and handling of hazardous drugs as outlined by U.S. Pharmacopeia chapters 797 and $800(4,5)$ and the Food and Drug Administration (๑). Investigators learned that IV flush bags containing saline, heparin, vancomycin, and ceftazidime had been compounded under substandard conditions, stored in a refrigerator, and accessed daily for multiple patients over approximately $4-8$ weeks until the solution was depleted.

Upon issuance of an order by the commissioner of the New York City Department of Health and Mental Hygiene on May 31, 2016, the provider ceased treating patients at clinic A until it became compliant with medication preparation and infection prevention standards. This outbreak highlights the gaps in both awareness and enforcement of national and state pharmacy and infection control standards in outpatient settings that perform parenteral medication compounding and infusion services (3-6). 


\section{Acknowledgments}

Marcelle Layton, Don Weiss, New York City Department of Health and Mental Hygiene; Nadine Shehab, Melissa Schaeffer, Division of Healthcare Quality Promotion, National Center for Emerging and Zoonotic Infectious Diseases, CDC; Brendan R. Jackson, Colleen Lysen, Joyce Peterson, Division of Foodborne, Waterborne, and Enteric Diseases, National Center for Emerging and Zoonotic Infectious Diseases, CDC.

\footnotetext{
${ }^{1}$ Epidemic Intelligence Service, CDC; ${ }^{2}$ Division of Healthcare Quality Promotion, National Center for Emerging and Zoonotic Infectious Diseases, CDC; ${ }^{3}$ New York City Department of Health and Mental Hygiene; ${ }^{4}$ Division of Foodborne, Waterborne, and Enteric Diseases, National Center for Emerging and Zoonotic Infectious Diseases, CDC; ${ }^{5}$ Laboratory Leadership Service, CDC; ${ }^{6}$ New York State Department of Health; ${ }^{7}$ Lenox Hill Hospital, Northwell Health System, New York, New York; ${ }^{8}$ Northwell Health Laboratories, Northwell Health System.
}

Corresponding author: Amber M. Vasquez, avasquez@cdc.gov, 404-718-1613.

\section{References}

1. Nachman S, Alpan O, Malowitz R, Spitzer ED. Catheter-associated fungemia due to Wangiella (Exophiala) dermatitidis. J Clin Microbiol 1996;34:1011-3.

2. CDC. Exophiala infection from contaminated injectable steroids prepared by a compounding pharmacy_United States, July-November 2002. MMWR Morb Mortal Wkly Rep 2002;51:1109-12.

3. CDC. Basic infection control and prevention plan for outpatient oncology settings. Atlanta GA: US Department of Health and Human Services, CDC; 2011. https://www.cdc.gov/hai/pdfs/guidelines/basic-infectioncontrol-prevention-plan-2011.pdf

4. US Pharmacopeial Convention, Inc. Chapter 797. Pharmaceutical compounding - sterile preparations. In: United States Pharmacopeia 39-National Formulary 34. Rockville, MD: US Pharmacopeial Convention, Inc.; 2016:626-70.

5. US Pharmacopeial Convention, Inc. Chapter 800. Hazardous drugshandling in healthcare settings. In: United States Pharmacopeia 39National Formulary 34, First supplement. Rockville, MD: US Pharmacopeial Convention, Inc.; 2016:7721-39.

6. Food and Drug Administration. Pharmacy compounding of human drug products under section 503A of the Federal Food, Drug, and Cosmetic Act. Silver Spring, MD: US Department of Health and Human Services, Food and Drug Administration; 2016. http://www.fda.gov/downloads/ Drugs/GuidanceComplianceRegulatoryInformation/Guidances/ UCM469119.pdf 